\title{
High-charge ultrashort electron bunch generation by an energy chirping cell-attached rf electron gun and its measurement using a transverse deflecting cavity
}

\author{
Y. Koshiba $\odot,{ }^{1, *}$ Y. Tadenuma $\odot,{ }^{1}$ S. Otsuka, ${ }^{1}$ M. Washio, ${ }^{1}$ \\ T. Takatomi, ${ }^{2}$ J. Urakawa ${ }^{2}{ }^{2}$ and K. Sakaue $\oplus^{1,3, \dagger}$ \\ ${ }^{1}$ Waseda Research Institute for Science and Engineering, Waseda University, \\ 3-4-1 Okubo, Shinjuku, Tokyo 169-8555, Japan \\ ${ }^{2}$ High Energy Accelerator Research Organization (KEK), 1-1 Oho, Tsukuba, Ibaraki 305-0801, Japan \\ ${ }^{3}$ Photon Science Center, The University of Tokyo, 7-3-1 Hongo, Bunkyo, Tokyo 113-8656, Japan
}

(Received 3 September 2020; revised 28 May 2021; accepted 15 July 2021; published 5 August 2021)

\begin{abstract}
Ultrashort electron bunches are becoming increasingly important for applications such as free-electron lasers, time-resolved electron diffraction/microscopy, and advanced accelerator concepts. We report femtosecond electron bunch generation with a unique rf gun and its precise measurement using a transverse deflecting cavity. A bunch length of $323 \pm 13$ fs with $120 \mathrm{pC}\left(\sim 7.5 \times 10^{8}\right.$ electrons) was achieved at a distance of just $3 \mathrm{~m}$ from the cathode. A longitudinal phase space distribution was also achieved, and we successfully experimentally confirmed the linearly energy chirped electron bunch generation and selfcompression by the velocity difference.
\end{abstract}

DOI: 10.1103/PhysRevAccelBeams.24.083401

\section{INTRODUCTION}

Photocathode rf electron guns have become a promising electron source for generating high-brightness electron beams. Electron bunches with low emittance and a picosecond bunch length are easily achieved with the help of commercialized mode-locked lasers because the initial conditions can be controlled by the laser pulse. This method has been adopted for $\mathrm{x}$-ray free-electron lasers (XFELs) [1-3], THz radiation sources [4-7], laserCompton X-ray sources [8-13], pulse radiolysis experiments [14-17], ultrafast electron diffraction/microscopy (UED/UEM) [18-23], and so on. For both these applications and future colliders, an ultrashort bunch length $(<1 \mathrm{ps})$ is of significant interest [24,25]. The temporal resolution can be improved in pump-probe experiments [26] and the coherent component in $\mathrm{THz}$ sources can be increased drastically [27]. Ultrashort bunches are also useful to probe the plasma wakefield, which is an advanced accelerating scheme with a very high-accelerating gradient [28]. For x rays, ultrashort pulses below 100 fs have already been achieved at XFEL facilities [2,29-33]. Moreover, this technology is progressing toward the attosecond regime

\footnotetext{
*koshibay@aoni.waseda.jp

ksakaue@psc.t.u-tokyo.ac.jp
}

Published by the American Physical Society under the terms of the Creative Commons Attribution 4.0 International license. Further distribution of this work must maintain attribution to the author(s) and the published article's title, journal citation, and DOI.
[34,35]. These methods have allowed new science and provided new knowledge in protein crystallography [36,37], photosynthesis [38], ablation studies [39,40], etc. In large accelerator facilities, it is common to arrange bunching systems to achieve ultrashort bunch lengths. Velocity bunching by off-crest acceleration and magnetic bunch compression are widely used [41,42]. In fact, all existing XFEL photoinjectors take advantage of several magnetic chicane bunch compressors to achieve high-density electron bunching and x-ray lasing $[43,44]$. Although an ultrashort bunch can be generated directly by irradiating a photocathode with a femtosecond laser pulse, this approach is successful only when the bunch charge is a few $\mathrm{pC}$ at most [45]. The severe space charge effect, especially in the lowenergy region near the cathode, causes bunch lengthening.

We have been conducting studies based on an $S$-band photocathode rf electron gun [46]. The rf electron gun cavity is based on the structure developed at Brookhaven National Laboratory [47], but some improvements have been made [48]. To overcome the difficulty of generating a highly charged $(\sim 100 \mathrm{pC})$ ultrashort electron bunch directly by a rf electron gun, we have proposed a unique cavity structure [49]. It has an extra cell coupled to the conventional 1.6-cell structure. The roll of the cell provides a linear energy chirp to the electron bunch. Thus, we have named the device as an energy chirping cell-attached rf gun (ECC-rf gun). In our early studies, we estimated the bunch length by analyzing the coherent $\mathrm{THz}$ radiation from the electron bunch and concluded that the bunch length was compressed to $<500 \mathrm{fs}$ rms $[49,50]$. For a more precise measurement, we designed and fabricated a transverse deflecting cavity (TDC) [51,52]. 
In this paper, we briefly explain the ECC-rf gun itself and the principle of the bunch length measurement using the TDC. Afterward, we show the experimental results of the ultrashort bunch length measurement and the longitudinal phase space measurement.

\section{ECC-RF GUN}

The ECC-rf gun is a photocathode rf gun specifically designed for ultrashort bunch generation [49]. A schematic of the cavity structure and the principle of operation is shown in Fig. 1.

The phase spaces are generated by general particle tracer (GPT) calculations [53]. The cavity is based on the conventional 1.6-cell design but also includes an ECC as an extra cell. The idea of adding an extra short cell was also studied at University of California, Los Angeles [54], for improving the temporal resolution of UED. The cavity structure such as the length of the short iris and the ECC was determined by iteration of modeling on SUPERFISH and beam tracking on GPT [49]. The electrons emitted from the photocathode are first accelerated up to approximately $4 \mathrm{MeV}$ and the space charge effect is mitigated. These relativistic electrons acquire a positive linear energy chirp at the adjacent ECC. Since the $\beta$ value, the velocity over light speed, of a $4 \mathrm{MeV}$ electron is 0.9918 , the energy difference still corresponds to the velocity difference. As a result, a few meters of drift space are sufficient for velocity bunching. As a design value predicted by GPT calculations, a bunch length of $88.4 \mathrm{fs}$ (rms) was expected at a distance of $230 \mathrm{~cm}$ from the cathode with a beam energy of 4.24 MeV. The design concept and calculation details are explained in Ref. [49]. The parameters of the experimental condition are shown in Table I.

\section{(a) Phase space}

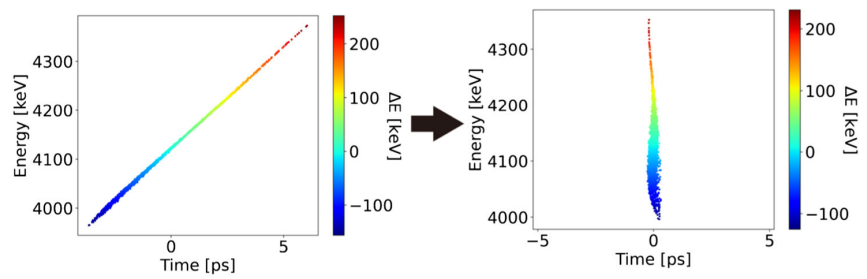

(b) Cavity structure and principle

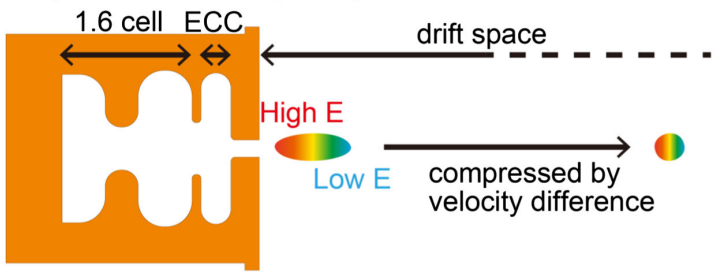

FIG. 1. (a) Phase space of the electron bunch generated by the ECC-rf gun simulated by GPT calculations. The colors show the energy difference. (b) Cavity structure and principle of operation of the ECC-rf gun.
TABLE I. Parameters of the experimental condition.
Cathode material

Charge [pC]

Laser wavelength $[\mathrm{nm}]$

Laser pulse energy $[\mu \mathrm{J}]$

Laser pulse width [ps, rms]

RF power to the gun [MW]

RF pulse width $[\mu \mathrm{s}]$

Repetition rate [Hz]

Electric field on cathode $[\mathrm{MV} / \mathrm{m}]$

Beam energy $[\mathrm{MeV}]$
CsTe

120

262 (4th harmonic of Nd:YLF)

$<8$

4

$<9.25$

2.0

5

77

3.8

\section{BUNCH LENGTH MEASUREMENT}

\section{A. Principle}

Several schemes have been established to measure short bunch lengths, such as radiation pulse duration detection by using streak cameras [55,56], spectral measurement of coherent radiation [57-60], bunch electric field detection by using the electro-optic effect $[58,61,62]$, and TDCs $[58,63,64]$. TDCs provide the most direct measurement of the electron bunch length. Our TDC is designed to measure the ultrashort bunch generated by the ECC-rf gun [51]. Table II shows the parameters related to our TDC.

A schematic of the principle of operation is shown in Fig. 2.

The $\mathrm{TM}_{210}$ magnetic field inside the TDC projects the longitudinal profile of the electron bunch onto the phosphor screen. By selecting zero-crossing rf phase and assuming a Gaussian distribution, the bunch length is expressed as [51]

$$
\sigma_{t}=\frac{p c}{e V_{\mathrm{T}} \omega L} \sqrt{\sigma_{\mathrm{on}}^{2}-\sigma_{\mathrm{off}}^{2}}=\frac{1}{\omega T} \sqrt{\sigma_{\mathrm{on}}^{2}-\sigma_{\mathrm{off}}^{2}},
$$

where $V_{\mathrm{T}}$ is the deflecting voltage, $\omega$ is the angular $\mathrm{rf}, L$ is the drift length from the TDC to the phosphor screen, $p$ is the momentum in the longitudinal direction, and $\sigma_{\text {on }}$ and $\sigma_{\text {off }}$ are the horizontal beam sizes when the TDC is on and off, respectively. $T=e V_{\mathrm{T}} L / p c$ is called the skewing strength and can be calibrated by measuring the deflection of the bunch centroid as a function of the rf phase. Concerning the temporal resolution, $\sigma_{\text {off }}$ should be as small as possible. The temporal resolution is defined by $\sigma_{\text {off }}$ as [51]

$$
\sigma_{\text {tres }}=\frac{p c}{e V_{T} \omega L} \sigma_{\text {off }}=\frac{1}{\omega T} \sigma_{\text {off }} .
$$

Note that the bunch length can be measured only where the TDC is placed.

TABLE II. Parameters of the TDC.

\begin{tabular}{lc}
\hline \hline RF frequency [MHz] & 2856 \\
RF power to the TDC [MW] & $<0.75$ \\
Deflecting voltage [MV] & $\approx 1$ \\
Drift space to phosphor screen [cm] & 74 \\
\hline \hline
\end{tabular}




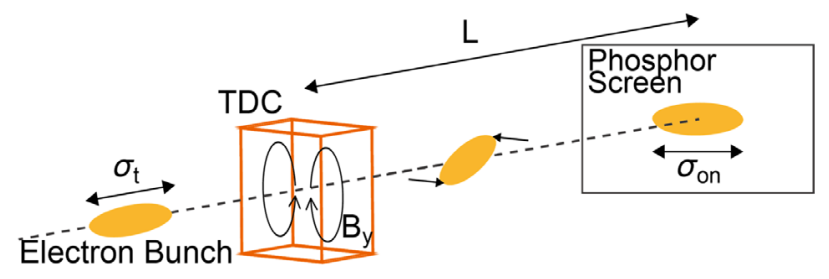

FIG. 2. Principle of the bunch length measurement. The vertical magnetic field streaks the electron bunch horizontally. TDC: transverse deflecting cavity.

\section{B. Experimental setup}

A schematic of the experimental setup is shown in Fig. 3.

The $262 \mathrm{~nm}, 4 \mathrm{ps}$ (rms) laser pulse, which is the fourth harmonic of the Nd:YLF mode-locked laser, is guided to the Cs-Te photocathode [65] almost perpendicularly by a holed mirror inside the beam pipe. The TDC was placed at three different distances from the cathode to measure the bunch length at different points: 155,223 , and $300 \mathrm{~cm}$. The waveguide was extended each time. The positions of quadrupole magnets and the phosphor screen relative to

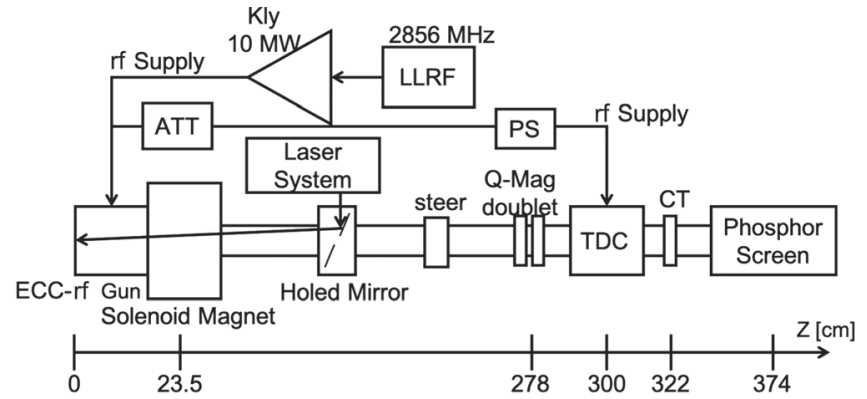

FIG. 3. Schematic of the experimental setup when the TDC is installed at $Z=300 \mathrm{~cm}$. The acronyms are as follows: LLRF: low-level rf, ATT: attenuator, PS: phase shifter, CT: current transformer, and TDC: transverse deflecting cavity.

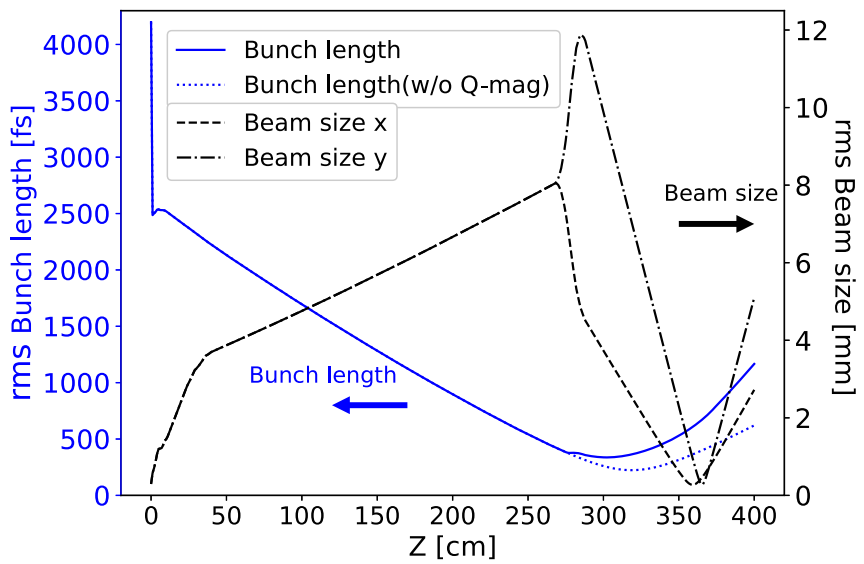

FIG. 4. Expected performance of the ECC-rf gun simulated by GPT. Expansion of the bunch length caused by space charge effect can be seen at $Z>270 \mathrm{~cm}$. the TDC was kept constant, so that the results could be simply compared. The drift space from the TDC to the phosphor screen was kept to $74 \mathrm{~cm}$. The input power and phase of the rf are controlled by the attenuator and the phase shifter. The maximum peak power of the klystron is $10 \mathrm{MW}$ and the maximum power provided to the TDC is $750 \mathrm{~kW}$. Since the klystron power is limited, the peak electric field at the cathode is limited to less than the ideal value of $100 \mathrm{MV} / \mathrm{m}$. The repetition rate of the $\mathrm{rf}$ is $5 \mathrm{~Hz}$. Using the values in Table I, GPT simulation indicates bunch length of $337 \mathrm{fs}$ at $Z=302 \mathrm{~cm}$, as shown in Fig. 4 .

\section{RESULTS}

First, we show the rf phase dependence of the charge and beam energy in Fig. 5.

An electron beam with a charge of $120 \mathrm{pC}$ and an energy of $3.8 \mathrm{MeV}$ is generated at a rf phase of $25^{\circ}$. As shown

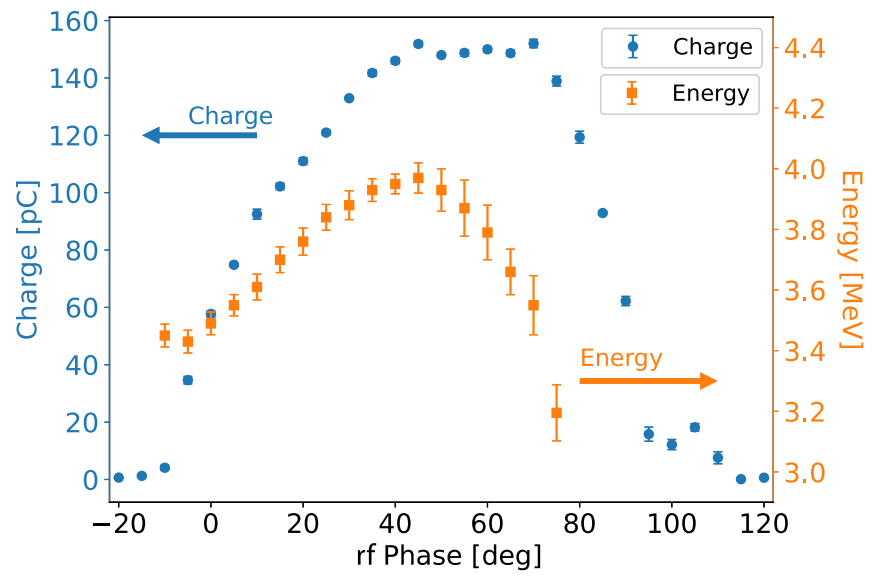

FIG. 5. Phase dependence of the charge and beam energy of the ECC-rf gun.
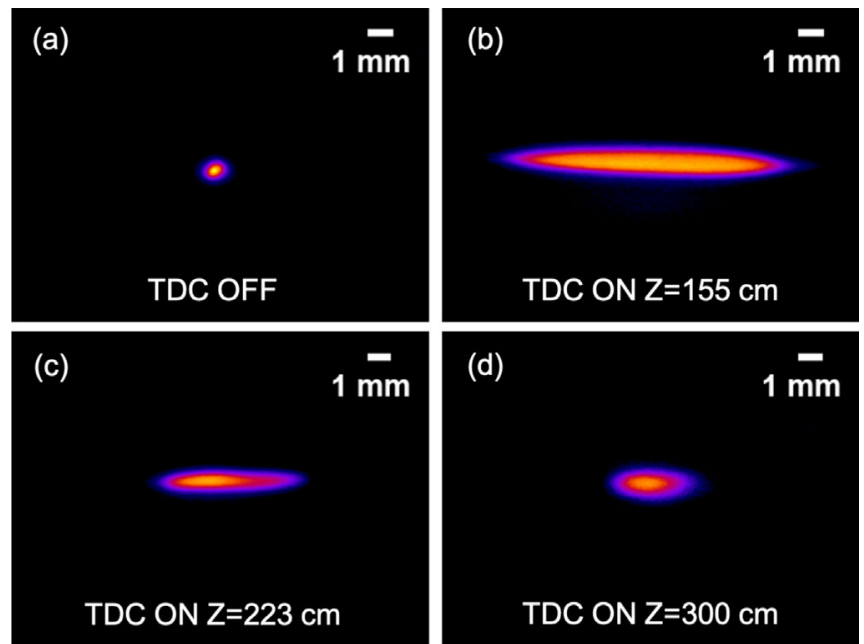

FIG. 6. (a) Example of a nondeflected beam profile. Deflected beam profiles when the TDC is placed at (b) $Z=155 \mathrm{~cm}$, (c) $Z=223 \mathrm{~cm}$, and (d) $Z=300 \mathrm{~cm}$. 
TABLE III. Measured bunch lengths.

\begin{tabular}{lccc}
\hline \hline $\mathrm{Z}[\mathrm{cm}]$ & 155 & 223 & 300 \\
$\sigma_{t}[\mathrm{fs}]$ & $2027 \pm 70$ & $694 \pm 46$ & $323 \pm 13$ \\
\hline \hline
\end{tabular}

below, the electron bunch is most compressed at this phase. The deflected beam profiles when the TDC is placed at $Z=155, Z=223$, and $Z=300 \mathrm{~cm}$ are shown in Fig. 6 . The off profile is an example of the TDC positioned at $Z=155 \mathrm{~cm}$.

A smaller horizontal beam size downstream indicates a shorter bunch length. By analyzing these images, the bunch length can be determined using Eq. (1). The bunch lengths at different points are summarized in Table III.

The bunch lengths were obtained from the average of five beam profile images. The numbers after \pm are standard deviation. We successfully confirmed the bunching effect of the ECC-rf gun. The minimum bunch length obtained was $323 \mathrm{fs}$ at $Z=300 \mathrm{~cm}$ and $120 \mathrm{pC}$. The bunch length with respect to the rf phase at three different positions is shown in Fig. 7.

Note that the experimental value of the charge is not kept constant in Fig. 7. They vary as shown in Fig. 5. Our TDC is composed of two cells and the total cell length is $73.04 \mathrm{~mm}$. The measured bunch length is the average within this range. One concern is the bunch lengthening caused by the transverse focus achieved by the quadrupole magnets. As shown in Fig. 4, GPT shows 224 fs at $Z=$ $319 \mathrm{~cm}$ without quadrupole magnets. 113 fs bunch lengthening and shift of the most compressed position occur due to the space charge effect.

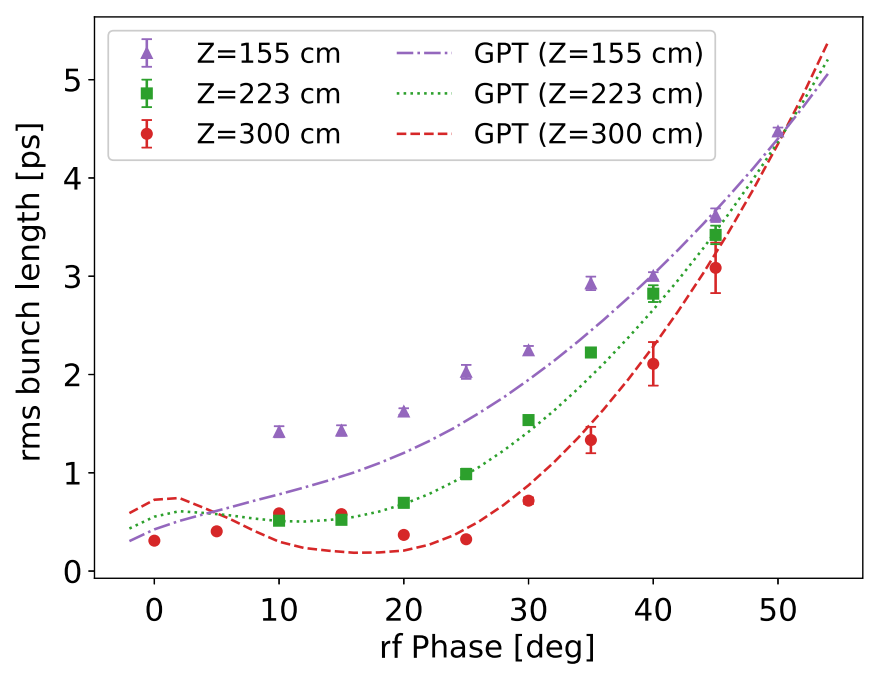

FIG. 7. Measured bunch lengths at three different positions as a function of the rf-accelerating phase. The dashed lines represent GPT simulations with a bunch charge of $100 \mathrm{pC}$.

\section{PHASE SPACE MEASUREMENT}

The principle for measuring the longitudinal phase space distribution is depicted in Fig. 8.

The combination of the TDC and the bending magnet enables us to visualize the longitudinal phase space distribution to the alumina phosphor screen. The TDC

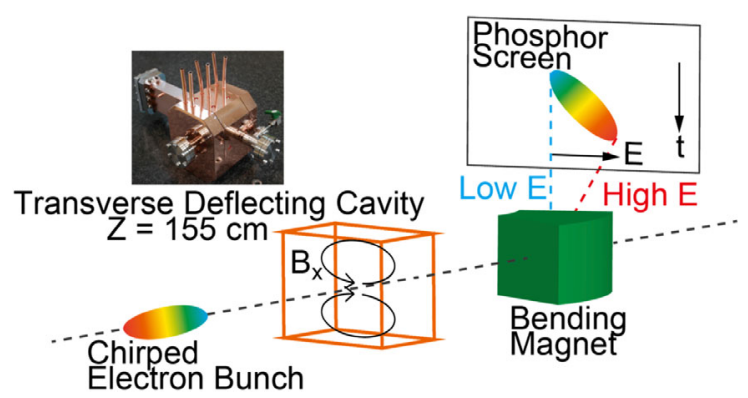

FIG. 8. Schematic of the longitudinal phase space measurement. The horizontal magnetic field in the TDC streaks the electron bunch vertically and the bending magnet disperses the electrons horizontally.
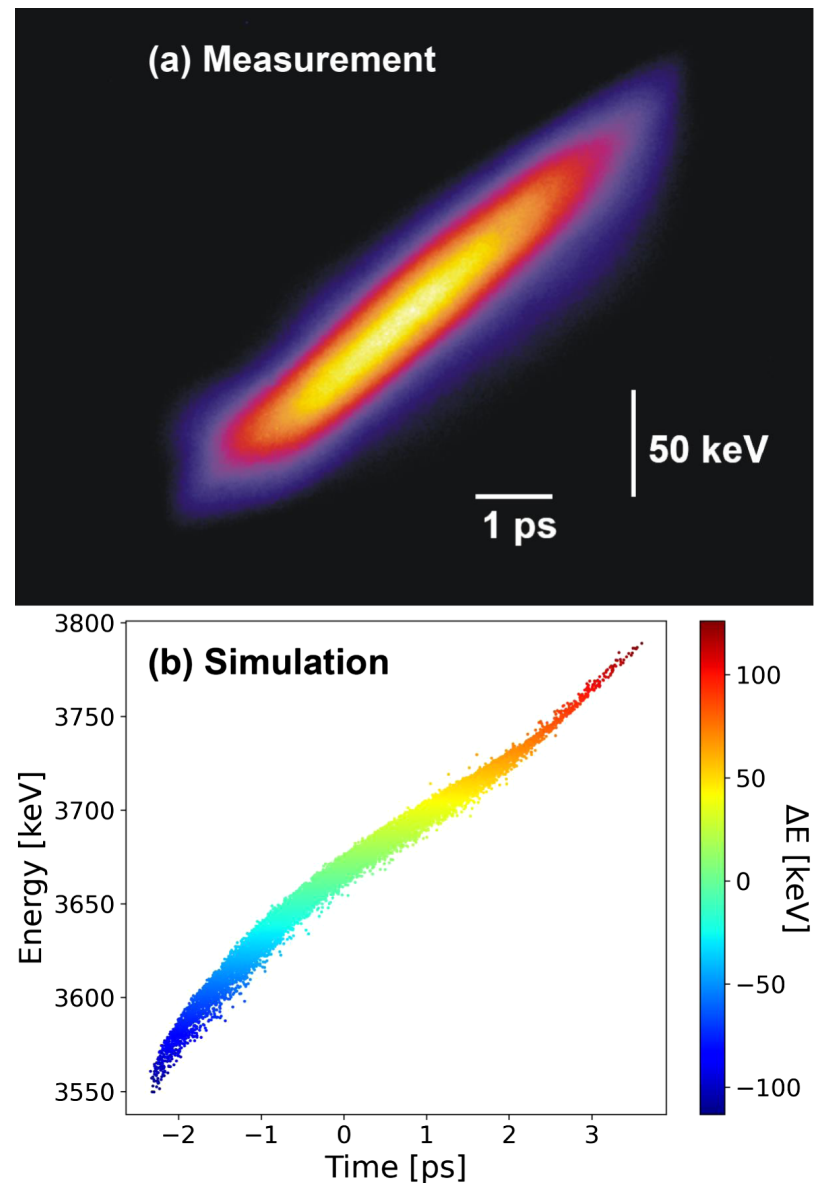

FIG. 9. Longitudinal phase space distribution obtained by (a) measurement with the TDC and a bending magnet and (b) GPT simulation at the position of $Z=155 \mathrm{~cm}$. The slopes of the linear chirp are (a) $27.4 \mathrm{keV} / \mathrm{ps}$ and (b) $35.0 \mathrm{keV} / \mathrm{ps}$. 
stretches the temporal profile vertically, and the bending magnet disperses the electrons horizontally according to their kinetic energy. Note that the TDC was rotated $90^{\circ}$ compared to the bunch length measurement setup. The experimental results and with the GPT simulation are shown in Fig. 9.

The image in Fig. 9(a) is the average of five images. The standard deviation of the centroid position, which corresponds to the bunch arrival timing jitter, is $127 \mathrm{fs}$. The bunch length jitter is $25 \mathrm{fs}$. The main factor of the jitter is the photocathode drive laser. We have successfully confirmed the generation of a linearly chirped electron bunch from the ECC-rf gun, which is required for effective velocity bunching. The slope of the energy chirp was determined to be $27.4 \mathrm{keV} / \mathrm{ps}$ experimentally and $35.0 \mathrm{keV} / \mathrm{ps}$ by GPT simulation. The experimental results were consistent with the simulation results in terms of not only the phase space measurement but also the bunch length measurement. We have clarified the proper operation of the ECC.

\section{SUMMARY}

Ultrashort electron bunch generation by the ECC-rf gun was performed and evaluated by direct measurement using a TDC. In our previous study, we estimated the bunch length by measuring coherent $\mathrm{THz}$ radiation and concluded that the bunch length was less than 500 fs [49]. In this study, we conducted a precise measurement using a TDC. We measured the bunch length at three different positions and successfully confirmed the velocity bunching. The minimum value demonstrated was $323 \pm 13$ fs at a distance of only $3 \mathrm{~m}$ from the photocathode. Furthermore, one can even shorten the distance by introducing a chicane. It should be highlighted that this achievement was obtained at $120 \mathrm{pC}$, which is an order of magnitude higher than the general bunch charge. It is expected that if we could feed the ECC-rf gun with a more powerful rf source, an even shorter bunch length and shorter distance could be achieved. Simulations with $110 \mathrm{MV} / \mathrm{m}$ at the cathode, which corresponds to a klystron power of $23 \mathrm{MW}$, indicate a bunch length of $127 \mathrm{fs}$ at $Z=264 \mathrm{~cm}$. We have also measured the longitudinal phase space distribution with the TDC and a bending magnet. The linear energy chirp was confirmed with good agreement to the simulation results.

In summary, we have demonstrated the generation of a highly charged ultrashort electron bunch by means of an ECC-rf gun. Applications such as UED/UEM, femtosecond pump-probe experiments, and $\mathrm{THz}$ radiation sources can be expected. Injection into an undulator would be an interesting experiment for intense $\mathrm{THz}$ generation.

\section{ACKNOWLEDGMENTS}

This work was supported by JSPS KAKENHI KAKENHI Grant-in-Aid for Young Scientists (B) 23740203 and Scientific Research (B) 18H01233.
[1] R. Akre et al., Phys. Rev. ST Accel. Beams 11, 030703 (2008).

[2] P. Emma et al., Nat. Photonics 4, 641 (2010).

[3] E. Prat, M. Aiba, S. Bettoni, B. Beutner, S. Reiche, and T. Schietinger, Phys. Rev. ST Accel. Beams 17, 104401 (2014).

[4] R. Kuroda, M. Yasumoto, H. Toyokawa, N. Sei, M. Koike, and K. Yamada, Nucl. Instrum. Methods Phys. Res., Sect. A 637, S30 (2011).

[5] S. Krainara, S. Chatani, H. Zen, T. Kii, and H. Ohgaki, Particles 1, 238 (2018).

[6] K. Kan, J. Yang, A. Ogata, T. Kondoh, M. Gohdo, H. Shibata, and Y. Yoshida, Electron. Commun. Jpn. 99, 22 (2016).

[7] Y. Tadenuma, T. Murakami, M. Brameld, M. Washio, K. Sakaue, R. Kuroda, and Y. Taira, J. Instrum. 15, C04016 (2020).

[8] I. Pogorelsky et al., Phys. Rev. ST Accel. Beams 3, 090702 (2000).

[9] K. Sakaue et al., Rev. Sci. Instrum. 80, 123304 (2009).

[10] R. Kuroda, H. Toyokawa, M. Yasumoto, H. IkeuraSekiguchi, M. Koike, K. Yamada, T. Yanagida, T. Nakajyo, F. Sakai, and K. Mori, Nucl. Instrum. Methods Phys. Res., Sect. A 637, S183 (2011).

[11] W. Graves et al., Phys. Rev. ST Accel. Beams 17, 120701 (2014).

[12] Y. Sakai, I. Pogorelsky, O. Williams, F. O'Shea, S. Barber, I. Gadjev, J. Duris, P. Musumeci, M. Fedurin, and A. Korostyshevsky, Phys. Rev. ST Accel. Beams 18, 060702 (2015).

[13] C. Vaccarezza et al., Nucl. Instrum. Methods Phys. Res., Sect. A 829, 237 (2016).

[14] J.F. Wishart, A. R. Cook, and J. R. Miller, Rev. Sci. Instrum. 75, 4359 (2004).

[15] J. L. Marignier, V. De Waele, H. Monard, F. Gobert, J. P. Larbre, A. Demarque, M. Mostafavi, and J. Belloni, Radiat. Phys. Chem. 75, 1024 (2006).

[16] Y. Hosaka, R. Betto, K. Sakaue, R. Kuroda, S. Kashiwagi, K. Ushida, and M. Washio, Radiat. Phys. Chem. 84, 10 (2013).

[17] J. Yang, K. Kan, M. Gohdo, and Y. Yoshida, in Novel Imaging and Spectroscopy, edited by J. Yang (IntechOpen, 2020), https://doi.org/10.5772/intechopen.91691.

[18] S. Weathersby et al., Rev. Sci. Instrum. 86, 073702 (2015).

[19] P. Zhu et al., New J. Phys. 17, 063004 (2015).

[20] J. Maxson, D. Cesar, G. Calmasini, A. Ody, P. Musumeci, and D. Alesini, Phys. Rev. Lett. 118, 154802 (2017).

[21] J. P. F. Nunes et al., Struct. Dyn. 7, 024301 (2020).

[22] F. Qi et al., Phys. Rev. Lett. 124, 134803 (2020).

[23] J. Yang, Y. Yoshida, and H. Yasuda, Microscopy 67, 291 (2018).

[24] T. Behnke, J. E. Brau, B. Foster, J. Fuster, M. Harrison, J. M. Paterson, M. Peskin, M. Stanitzki, N. Walker, and H. Yamamoto, The international linear collider technical design report—volume 1: executive summary, arxiv:1306.6327.

[25] M. Aicheler, P. Burrows, M. Draper, T. Garvey, P. Lebrun, K. Peach, N. Phinney, H. Schmickler, D. Schulte, and N. Toge, A multi-TeV linear collider based on CLIC technology: CLIC conceptual design report, Report No. CERN-2012-007, CERN, 2012. 
[26] T. Kondoh, J. Yang, K. Norizawa, K. Kan, T. Kozawa, A. Ogata, S. Tagawa, and Y. Yoshida, Radiat. Phys. Chem. 84, 30 (2013).

[27] T. Nakazato et al., Phys. Rev. Lett. 63, 1245 (1989).

[28] Y. Sakai et al., Phys. Rev. Accel. Beams 21, 101301 (2018).

[29] W. Ackermann et al., Nat. Photonics 1, 336 (2007).

[30] M. Yabashi, H. Tanaka, and T. Ishikawa, J. Synchrotron Radiat. 22, 477 (2015).

[31] T. Ishikawa et al., Nat. Photonics 6, 540 (2012).

[32] H. S. Kang et al., Nat. Photonics 11, 708 (2017).

[33] E. Prat, P. Dijkstal, E. Ferrari, and S. Reiche, Phys. Rev. Lett. 124, 074801 (2020).

[34] S. Huang, Y. Ding, Y. Feng, E. Hemsing, Z. Huang, J. Krzywinski, A. Lutman, A. Marinelli, T. Maxwell, and D. Zhu, Phys. Rev. Lett. 119, 154801 (2017).

[35] P. K. Maroju et al., Nature (London) 578, 386 (2020).

[36] M. Suga et al., Nature (London) 543, 131 (2017).

[37] K. Yamashita et al., Sci. Rep. 5, 14017 (2015).

[38] J. Kern et al., Nat. Commun. 5, 4371 (2014).

[39] T. Shibuya et al., Appl. Phys. Lett. 113, 171902 (2018).

[40] T. H. Dinh et al., Commun. Phys. 2, 150 (2019).

[41] D. Filippetto et al., Phys. Rev. ST Accel. Beams 14, 092804 (2011).

[42] F. Zhou, K. Bane, Y. Ding, Z. Huang, H. Loos, and T. Raubenheimer, Phys. Rev. ST Accel. Beams 18, 050702 (2015).

[43] T. Inagaki, C. Kondo, H. Maesaka, T. Ohshima, Y. Otake, T. Sakurai, K. Shirasawa, and T. Shintake, Phys. Rev. ST Accel. Beams 17, 080702 (2014).

[44] S. Bettoni, M. Pedrozzi, and S. Reiche, Phys. Rev. ST Accel. Beams 18, 123403 (2015).

[45] Y. Murooka, N. Naruse, S. Sakakihara, M. Ishimaru, J. Yang, and K. Tanimura, Appl. Phys. Lett. 98, 251903 (2011).

[46] K. Sakaue, H. Hayano, S. Kashiwagi, R. Kuroda, A. Masuda, T. Suzuki, T. Takatomi, N. Terunuma, J. Urakawa, and M. Washio, Nucl. Instrum. Methods Phys. Res., Sect. B 269, 2928 (2011).

[47] X. J. Wang and I. Ben-Zvi, in Proceedings of the Particle Accelerator Conference Vancouver, BC, Canada, 1997 (IEEE, New York, 1997), p. 2793.

[48] A. Deshpande, S. Araki, M. Fukuda, K. Sakaue, N. Terunuma, J. Urakawa, N. Sasao, and M. Washio, Nucl. Instrum. Methods Phys. Res., Sect. A 600, 361 (2009).
[49] K. Sakaue, Y. Koshiba, M. Mizugaki, M. Washio, T. Takatomi, J. Urakawa, and R. Kuroda, Phys. Rev. ST Accel. Beams 17, 023401 (2014).

[50] Y. Koshiba, K. Sakaue, M. Mizugaki, M. Washio, R. Kuroda, T. Takatomi, and J. Urakawa, Vib. Spectrosc. 75, 184 (2014).

[51] Y. Nishimura, K. Sakaue, M. Nishiyama, T. Takahashi, M. Washio, T. Takatomi, and J. Urakawa, Nucl. Instrum. Methods Phys. Res., Sect. A 764, 291 (2014).

[52] K. Sakaue, Y. Nishimura, M. Nishiyama, T. Takahashi, M. Washio, T. Takatomi, and J. Urakawa, Jpn. J. Appl. Phys. 54, 026301 (2015).

[53] S. B. van der Geer and M. J. de Loos, in Proceedings of 1998 European Particle Accelerator Conference (EPAC'98), Stockholm, Sweden (JACoW, Stockholm, 1998), p. 1245.

[54] P. Musumeci, L. Faillace, A. Fukasawa, J. T. Moody, B. O'Shea, J. B. Rosenzweig, and C. M. Scoby, Microsc. Microanal. 15, 290 (2009).

[55] J. Yang, T. Kondoh, K. Kan, T. Kozawa, Y. Yoshida, and S. Tagawa, Nucl. Instrum. Methods Phys. Res., Sect. A 556, 52 (2006).

[56] C. Chen et al., J. Phys. Conf. Ser. 1067, 072013 (2018).

[57] P. Kung, H. C. Lihn, H. Wiedemann, and D. Bocek, Phys. Rev. Lett. 73, 967 (1994).

[58] Y. Otake, Nucl. Instrum. Methods Phys. Res., Sect. A 637, S7 (2011).

[59] M. Veronese, R. Appio, P. Craievich, and G. Penco, Phys. Rev. Lett. 110, 074802 (2013).

[60] I. Nozawa et al., Phys. Rev. ST Accel. Beams 17, 072803 (2014).

[61] G. Berden et al., Phys. Rev. Lett. 99, 164801 (2007).

[62] S. Casalbuoni, H. Schlarb, B. Schmidt, P. Schmüser, B. Steffen, and A. Winter, Phys. Rev. ST Accel. Beams 11, 072802 (2008).

[63] P. Emma, J. Frisch, and P. Krejcik, Linear collider collaboration tech notes, Technical Report No. LCLSTN-00-12 (SLAC-PUB-8864), 2000.

[64] H. Ego, H. Maesaka, T. Sakurai, Y. Otake, T. Hashirano, and S. Miura, Nucl. Instrum. Methods Phys. Res., Sect. A 795, 381 (2015).

[65] T. Tamba, J. Miyamastsu, K. Ezawa, Y. Koshiba, K. Sakaue, H. Ijima, and M. Washio, J. Instrum. 15, C05006 (2020). 